Review

\title{
Associations of delta fatty acid desaturase gene polymorphisms with lipid metabolism disorders
}

\author{
Oksana Yu. Kytikova, Tatyana P. Novgorodtseva, Yulia K. Denisenko, Marina V. Antonyuk, Tatyana A. Gvozdenko \\ Vladivostok Branch of Far Eastern Scientific Center of Respiratory Physiology and Pathology, Research Institute of Medical Climatology and \\ Rehabilitative Treatment, Vladivostok, Russia
}

Received 6 October 2020, Revised 14 February 2021, Accepted 2 August 2021

(C) 2020, Russian Open Medical Journal

\begin{abstract}
Overweight, obesity, type 2 diabetes mellitus, metabolic syndrome, cardiovascular diseases, and non-alcoholic fatty liver disease are common chronic ailments associated with lipid metabolism disorders. One of the mechanisms of these disorders is related to the deficiency and/or change in the balance of essential fatty acids (FAs). At the same time, the provision of $\omega 3$ and $\omega 6$ polyunsaturated fatty acids (PUFAs) depends, besides sufficient dietary intake, on efficiency of their endogenous biosynthesis by desaturation and elongation processes regulated by FA elongases and FA desaturases. Desaturases are encoded by PUFA desaturase genes (FADSs): FADS1 and FADS2. Alteration of FA desaturase activity and single nucleotide polymorphisms (SNPs) in the FADS1 and FADS2 gene cluster are associated with lipid metabolism dysfunction and may affect the pathogenesis of lipid-related diseases. People of different ages, from different ethnic backgrounds and countries may exhibit varying degrees of response to dietary supplements of $\omega 3$ and $\omega 6$ PUFAs. The study of the relationship between lipid metabolism disorders and genetic factors controlling FA metabolism is an important research area since the health effects of alimentary $\omega 3$ and $\omega 6$ PUFAs can depend on genetic variants of the FADS genes. This review summarizes the literature data on the association of FADS gene polymorphisms with lipid metabolism disorders and their role in the development of chronic noncommunicable pathologies associated with changes in lipid metabolism.
\end{abstract}

Keywords: fatty acid, fatty acid desaturase gene, lipid metabolism.

Cite as Kytikova OYu, Novgorodtseva TP, Denisenko YuK, Antonyuk MV, Gvozdenko TA. Associations of delta fatty acid desaturase gene polymorphisms with lipid metabolism disorders. Russian Open Medical Journal 2021; 10: e0403.

Correspondence to Oksana Yu. Kytikova. Address: 73g Russkaya St., Vladivostok 690105, Russian Federation. Phone: +74232788201. E-mail: kytikova@yandex.ru.

\section{Introduction}

Polyunsaturated fatty acids (PUFAs) are components of the diet and are endogenously produced by the elongation and desaturation from essential fatty acids (FAs), such as $\alpha$-linolenic $(18: 3 \omega 3)$ and linoleic $(18: 2 \omega 6)$ acids [1]. The effect of PUFAs is associated with their activation via specific receptors, ability to modulate the function of membrane lipid rafts and activity of many signaling pathways, as well as with genetic and epigenetic mechanisms [2-4]. The study of $\omega 3$ and $\omega 6$ PUFA metabolites is of interest due to their involvement in immunometabolic responses and the pathogenesis of chronic diseases associated with lipid metabolism disorders $[5,6]$. The lack of essential FAs impairs the resolution of inflammation and results in the development of a slow chronic low-grade inflammation [7, 8]. This type of inflammation underlies many non-communicable chronic diseases and conditions accompanied by lipid metabolism dysfunction, in particular obesity, cardiovascular diseases (CVDs), type 2 diabetes mellitus (DM2), metabolic syndrome (MS), non-alcoholic fatty liver disease (NAFLD) [1, 8-11]. Obesity, overweight, MS, CVDs, and NAFLD are common in both adults and children [12, 13]. Childhood obesity is a risk factor for developing CVDs and DM2 in adulthood [14]. Their relationships with lipid metabolism disorders in pregnant and breast-feeding women is studied as well [15].
PUFAs are widely used as dietary supplements for the prevention and treatment of lipid metabolism pathologies [16-18]. Despite this, there are contradictory results regarding their effectiveness $[17,19,20]$. In the course of analyzing the available data, it is necessary to consider such factors as variable bioavailability of dietary $\omega 3$ PUFAs, background levels of $\omega 3$ and $\omega 6$ PUFAs, functional state of gut microbiota, and experimental conditions used in animal studies that are more homogeneous and controlled compared with studies involving humans $[8,21,22]$. Some studies confirm the metabolic and anti-inflammatory properties of $\omega 6$ PUFA, especially linoleic acid, while other publications emphasize that the conversion of this FA into arachidonic acid is accompanied by the formation of its proinflammatory metabolites - eicosanoids [23]. Such contradictory views and fragmented results can be explained by the fact that people of different ages, from different ethnic groups and countries, may exhibit different degrees of response to dietary PUFAs $[24,25]$. Children, pregnant and breast-feeding women have their own features of responding to the dietary PUFA supplementation [26-29].

The heterogeneity of the response to treatment may be associated with genetic variability among individuals $[20,30]$. Endogenous processing of PUFAs is controlled by FA elongases and FA desaturases encoded by PUFA desaturase (delta fatty acid 
desaturases, FADSS) genes [31]. Changes in the activity of FA desaturases and polymorphisms of their genes are observed in a significant number of diseases related to lipid metabolism dysregulation [32-34]. At the same time, the genetic regulation of endogenous lipid metabolism is poorly understood [35, 36]. Investigating the association of genetic factors controlling FA metabolism with lipid metabolism disorders and development of the low-grade inflammation in chronic non-communicable diseases can be considered as a promising study field. The health effects of dietary $\omega 3$ and $\omega 6$ PUFAs may vary with genetic variants within the FADS genes.

We carried out bibliographic searches in PubMed for reports of randomized controlled trials published between 2010 and 2020. In our search for relevant publications, the following keyword combinations were used: fatty acids, fatty acid desaturase, fatty acid desaturase genes, and lipid metabolism. Only articles in English were considered. Hence, 481 publications were identified, of which 19 were the reviews.

Our analysis of available literature established that international databases contained a sufficient number of the articles dedicated to studying the relationships between FA synthesis disorders and chronic diseases [1, 5-7, 21, 24, etc.], as well as enough publications highlighting the effects of alimentary PUFAs intake in various pathological conditions [8-10, 16-20, 22, 23,25 , etc.]. The articles discussing genetic regulation of endogenous lipid metabolism were less abundant [4, 11, 24-29, etc.]. There were just a few studies aimed at establishing the health effects of alimentary PUFAs depending on FADS genetic variants $[26,35,36$, etc.]. Finally, only a small number of the relevant literary sources were reviews, which implied the urgent need to systematize current accumulated data. It should be noted that there were some reviews summarizing the data on association of FADS gene polymorphisms with the development of diseases related to altered lipid metabolism [2].

In our review, we analyze literary sources of the last decade on the relationships between FADS gene polymorphisms, FA synthesis disorders and the development of socially significant chronic diseases associated with changes in lipid metabolism (overweight, obesity, DM2, MS, CVD, and NAFLD). The features of FADS genotype in adults, children, along with pregnant and breastfeeding women are described. The link between genetic factors controlling FA metabolism and lipid metabolism disorders in adults and children is defined. The therapeutic prospects of alimentary PUFA supplementation are discussed as well.

\section{Metabolism of $\omega-3$ and $\omega-6$ PUFAs}

\section{The $\omega-3$ and $\omega-6$ PUFAs}

Fatty acids are carboxylic acids with a carbon chain containing carboxyl and methyl groups at its ends [31]. Natural FAs are classified into saturated and unsaturated. In contrast to unsaturated FAs, saturated FAs do not have double bonds. If unsaturated FAs contain one double bond, they are called monoenic (monounsaturated) FAs; if they have from two to six double bonds, they are polyunsaturated fatty acids (PUFAs). PUFAs are classified into $\omega 9, \omega 7, \omega 6$, and $\omega 3$ FAs by the position of the first double bond closest to the methyl end of the carbon chain. Additionally, according to the length of the carbon chain (C), FAs are grouped into long-chain (C12-C22), medium-chain (C7-C12) and short-chain (C2-C6) categories. Medium- and short-chain FAs are synthesized de novo or are a result of fat consumption [37].
Short-chain FAs can be produced in the intestine during partial digestion of soluble fiber and then enter the circulatory system [38]. Some $\omega 3$ and $\omega 6$ PUFAs are essential and cannot be synthesized by the human organism; their sufficient intake is critical for its normal functioning $[39,40]$.

Synthesis of saturated FAs occurs in the cytoplasm of cells of the liver, kidneys, intestines, brain, lung, mammary glands, as well as in adipose tissue (with the participation of acetyl-CoA, NADPH, ATP, $\mathrm{Mn}^{2+}$ and $\mathrm{HCO}^{3-}$ ), while the lengthening of the carbon chain of FAs (both endogenous FAs synthesized in the cytoplasm, and exogenous FAs coming from the intestine) takes place in the mitochondria and involves $\beta$-oxidation reactions. Other FAs are formed from palmitic acid synthesized in the cell cytoplasm by elongation of the carbon chain (lengthening) and desaturation (formation of double bonds) with the participation of malonyl-CoA [41]. The processes of elongation and desaturation are repeated and combined with each other.

Enzymes involved in the biosynthesis of monounsaturated and polyunsaturated FAs are called FA desaturases. The transformation of saturated FAs into monounsaturated FAs is catalyzed by stearoyl-CoA-desaturase-1. In mammals, there are no enzymes catalyzing the introduction of double bonds into the FA carbon chain after the 9th carbon atom. Therefore, essential FAs ( $\alpha$ linolenic and linoleic) can be obtained exclusively with food [42, 43]. Linoleic acid is converted into FAs of the $\omega 6$ family: $\gamma$-linolenic

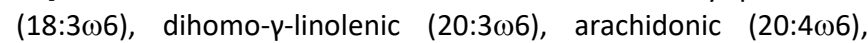

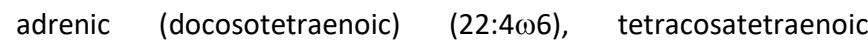

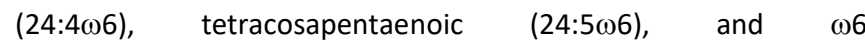
docosapentaenoic $(22: 5 \omega 6)$ acids. In turn, $\alpha$-linolenic acid is transformed into FAs of the $\omega 3$ family: stearidonic (18:4 $\omega 3)$,

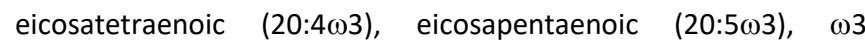

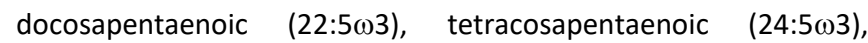
tetracosahexaenoic $(24: 6 \omega 3)$, and docosahexaenoic $(22: 6 \omega 3)$ (Table 1).

\section{The enzymes involved in metabolism of $\omega-3$ and $\omega-6$ PUFAs}

The synthesis of $\omega 6$ and $\omega 3$ PUFAs is regulated by the elongation and desaturation enzymes: elongase 2 (ELOVL2) and elongase 5 (ELOVL5), $\triangle 5$ desaturase (delta- 5 desaturase (D5D) or FADS1) and $\Delta 6$ desaturase (delta- 6 desaturase (D6D) or FADS2) $[44,45]$. Elongases are responsible for the lengthening of the carbon chain in PUFAs. Desaturases catalyze the conversion of a single bond between carbon atoms into a double bond $(C=C)$ called unsaturated bonds. The biosynthesis of docosahexaenoic (22:6 133$)$ and $\omega 6$ docosapentaenoic (22:5 $\omega 6$ ) acids includes the formation of FAs with 24 carbon atoms in the endoplasmic reticulum and their further movement to the center of $\beta$-oxidation. Considering that FAs can be a substrate for peroxisomal oxidation, they are involved in the regulation of FA composition of membrane lipids $[3,15]$.

PUFAs of the $\omega 6$ and $\omega 3$ families are synthesized under the influence of the same enzymes. Thus, as a result of the synthesis of long-chain $\omega 3$ PUFAs with the participation of FADS2, stearidonic acid $(18: 4 \omega 3)$ is formed from $\alpha$-linolenic acid $(18: 3 \omega 3)$ that is converted into eicosatetraenoic acid (20:403). FADS1 catalyzes the formation of eicosapentaenoic acid $(20: 5 \omega 3)$. Then, with the participation of ELOVL2, microsomal elongation of the $\omega 3$ chain of docosapentaenoic acid $(22: 5 \omega 3)$ to tetracosapentaenoic acid $(24: 5 \omega 3)$ occurs followed by desaturation at the position- 6 by FADS2 to tetracosahexaenoic acid (24:6ஸ3). Further, this PUFA is 
metabolized through $\beta$-oxidation in peroxisomes to docosahexaenoic acid (22:6ஸ3). Both $\alpha$-linolenic $(18: 3 \omega 3)$ and tetracosapentaenoic $(24: 5 \omega 3)$ acids can serve a substrate for desaturation at position- 6 of FAs by FADS2 [46].

The sequential synthesis of long-chain $\omega 6$ PUFAs begins with

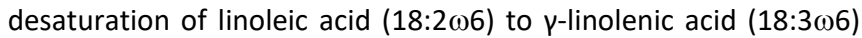
under the impact of FADS2. The resulting acid is metabolized by ELOVL5 into dihomo- $\gamma$-linolenic acid $(20: 3 \omega 6)$ that, in turn, is converted into arachidonic acid $(20: 4 \omega 6)$ by FADS1. Further

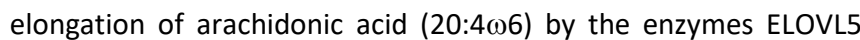
and ELOVL2 leads to the formation of adrenic (docosotetraenoic) $(22: 4 \omega 6)$ and tetracosatetraenoic $(24: 4 \omega 6)$ acids. At this stage, FADS2 lead to the transformation of tetracosatetraenoic acid $(24: 4 \omega 6)$ into tetracosapentaenoic $(24: 5 \omega 6)$, and then, as a result of $\beta$-oxidation, $\omega 6$ docosapentaenoic acid $(22: 5 \omega 6)$ is synthesized.

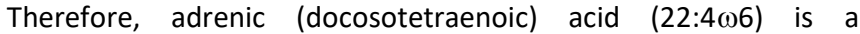
progenitor of $\omega 6$ docosapentaenoic acid $(22: 5 \omega 6)$, but this pathway does not depend on acyl-CoA-dependent $\Delta 4$ desaturase and requires moving between the endoplasmic reticulum and the center of $\beta$-oxidation.

The activity of FA desaturases is controlled by the level of dietary PUFAs intake, which can affect the FADS gene expression [47-49].

\section{An impact of polymorphisms of the FADS1 and FADS2 genes on the plasma lipid profile}

FA desaturases are encoded by genes FADS1 and FADS2, located on the long arm of the human chromosome 11 and on mouse chromosome 19 [45]. In addition to FADS1 and FADS2, other genes that can also affect the plasma level of $\omega 6$ PUFAs have been reported: genes on chromosome 10 associated with linoleic

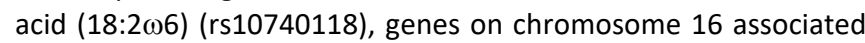
with linoleic acid, $\gamma$-linolenic $(18: 3 \omega 6)$, dihomo- $\gamma$-linolenic

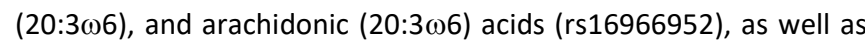

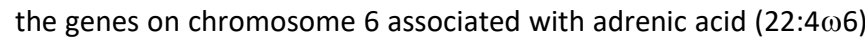
(rs3134950) [50]. It has been shown that single nucleotide polymorphisms (SNPs) - i.e., a single base change (A, T, G, C) in a DNA sequence of the FADS gene cluster - alter the activity of FA desaturases, plasma lipid profile and glucose homeostasis [51]. Close relationships between FADS1 and FADS2 gene polymorphisms, and the plasma levels of $\omega 3$ PUFAs, $\omega 6$ PUFAs, high-density lipoproteins (HDL), low-density lipoproteins (LDL), and triglycerides have been established [4, 52]. Polymorphisms of the FADS1 and FADS2 genes have a different effect on the plasma lipid profile in adults, children, as well as pregnant and breast-feeding women (Table 2).

Table 1. The spectrum of $\omega 3$ and $\omega 6$ fatty acids synthesized from $\alpha$-linolenic and linoleic acids

\begin{tabular}{|c|c|c|c|}
\hline \multicolumn{2}{|c|}{ The $\omega 6$ FAs synthesized from linoleic acid $(18: 2 \omega 6)$} & \multicolumn{2}{|c|}{ The $\omega 3$ FAs synthesized from $\alpha$-linolenic acid (18:3 $\omega 3)$} \\
\hline Name & Formula & Name & Formula \\
\hline p-linolenic acid & $18: 3 \omega 6$ & stearidonic acid & $18: 4 \omega 3$ \\
\hline dihomo- $\nu$-linolenic acid & $20: 3 \omega 6$ & eicosatetraenoic acid & $20: 4 \omega 3$ \\
\hline arachidonic acid & $20: 4 \omega 6$ & eicosapentaenoic acid & $20: 5 \omega 3$ \\
\hline adrenic acid (docosotetraenoic) acid & $22: 4 \omega 6$ & $\omega 3$ docosapentaenoic acid & $22: 5 \omega 3$ \\
\hline tetracosatetraenoic acid & $24: 4 \omega 6$ & tetracosapentaenoic acid & $24: 5 \omega 3$ \\
\hline tetracosapentaenoic acid & $24: 5 \omega 6$ & tetracosahexaenoic acid & $24: 6 \omega 3$ \\
\hline
\end{tabular}

Table 2. Associations of FADS1 and FADS2 gene polymorphisms with PUFA levels in adults, children, pregnant and breast-feeding women

\begin{tabular}{|c|c|c|}
\hline SNPS & Association with level of PUFAs & References \\
\hline \multirow{2}{*}{ FADS1-rs174546 } & plasma level of $\omega 3$ PUFAs in adults & [53] \\
\hline & plasma level of $\omega 3$ PUFAs in pregnant women & [54] \\
\hline FADS1-rs174547 & plasma level of $\omega 3$ PUFAs in adults & [53] \\
\hline \multirow{2}{*}{ FADS1-rs174550 } & plasma level of $\omega 3$ PUFAs in adults & [53] \\
\hline & plasma level of $\omega 6$ PUFAs in adults & {$[55]$} \\
\hline \multirow{2}{*}{ FADS1-rs174556 } & plasma level of $\omega 3$ and $\omega 6$ PUFAs in pregnant women & [56] \\
\hline & level of $\omega 6$ PUFAs in breast milk & [57] \\
\hline \multirow{2}{*}{ FADS2-rs1535 } & plasma level of $\omega 3$ PUFAs in adults & [53] \\
\hline & level of $\omega 6$ PUFAs in breast milk & [58] \\
\hline FADS2-rs174546 & plasma level of $\omega 3$ PUFAs in adults & [53] \\
\hline FADS2-rs174576 & plasma level of $\omega 3$ PUFAs in adults & [53] \\
\hline FADS2-rs174575 & $\begin{array}{l}\text { plasma level of } \omega 3 \text { PUFAs in children (girls and boys) } \\
\text { plasma level of } \omega 6 \text { PUFAs in boys }\end{array}$ & [59] \\
\hline FADS2-rs174577 & plasma level of $\omega 3$ PUFAs in adults & [53] \\
\hline FADS2-rs174602 & plasma level of $\omega 3$ and $\omega 6$ PUFAs in pregnant women & [56] \\
\hline \multicolumn{3}{|l|}{ FADS-rs174537 } \\
\hline FADS-rs174561 & plasma level of $\omega 3$ PUFAs in adults & {$[50]$} \\
\hline \multicolumn{3}{|l|}{ FADS-rs3834458 } \\
\hline \multicolumn{3}{|l|}{ Haplotypes } \\
\hline FADS2-rs1535-rs3834458 & level of $\omega 6$ PUFAs in breast milk & [58] \\
\hline FADS2-rs1535-rs174575 & level of $\omega 6$ PUFAs in breast milk & [58] \\
\hline FADS1-rs174547-rs174553 & level of $\omega 6$ PUFAs in breast milk & [58] \\
\hline FADS2 rs3834458-rs1535-rs174575 & level of $\omega 6$ PUFAs in breast milk & [58] \\
\hline
\end{tabular}


Table 3. Associations of FADS1 and FADS2 gene polymorphisms with lipid metabolism-related diseases

\begin{tabular}{|c|c|c|c|}
\hline SNPS & Association with parameters & Pathology & References \\
\hline FADS1-rs174547 & $\begin{array}{l}\text { waist circumference, fasting blood glucose, } \\
\text { plasma HDL level, BMI }\end{array}$ & MS, DM2 & {$[32,71,72]$} \\
\hline FADS2-rs 2727270 & fasting blood glucose & DM2 & [32] \\
\hline FADS2-rs 174575 & high birth weight & risk of developing metabolic disorders & [28] \\
\hline FADS2-rs174601 & serum lipid level & risk of developing IHD and IS & [73] \\
\hline FADS2-rs174602 & high birth weight & risk of developing metabolic disorders & [56] \\
\hline $\begin{array}{l}\text { FADS1-rs174545 } \\
\text { FADS1-rs174548 FADS1- } \\
\text { rs174553 FADS2-rs1535 } \\
\text { FADS2-rs174583 }\end{array}$ & BMI & risk of developing obesity in pregnancy & [29] \\
\hline FADS1-rs174546 & BMI, serum lipid level & $\begin{array}{l}\text { risk of developing obesity in pregnancy, } \\
\text { risk of developing IHD and IS }\end{array}$ & {$[29,73,74]$} \\
\hline FADS2-rs174601 T/T & serum lipid level & risk of developing IHD and IS & [73] \\
\hline FADS-rs174537G/T & - & high risk of developing IHD & {$[75,76]$} \\
\hline FADS-rs $174537 \mathrm{~T}$ & - & low risk of developing IHD & {$[75,76]$} \\
\hline FADS-rs174537 GG & DM2 & high risk of developing IHD & {$[75,76]$} \\
\hline Haplotypes & & & \\
\hline $\begin{array}{l}\text { FADS1-rs174546 } \\
- \text {-FADS2-rs174601 T/T) }\end{array}$ & serum lipid level & high risk of developing IS & [73] \\
\hline $\begin{array}{l}\text { FADS1-rs174546 } \\
\text {-FADS2-rs174601 C/C) }\end{array}$ & serum lipid level & low risk of developing IHD & [73] \\
\hline
\end{tabular}

SNP, single nucleotide polymorphism; MS, metabolic syndrome; IHD, ischemic heart disease; IS, ischemic stroke; DM2, type 2 diabetes mellitus; BMI, body mass index; HDL, high-density lipoproteins.

\section{An impact of polymorphisms in the FADS1 and FADS2 genes on levels of $\omega 3$ and $\omega 6$ PUFAs}

O. Coltell et al. established correlations between SNPs in the FADS gene cluster (FADS1-rs174546; FADS1-rs174547; FADS1rs174550; FADS2-rs1535; FADS2-rs174546; FADS2-rs174576; FADS2-rs174577) and plasma concentration of $\omega 3$ PUFAs [53]. M. Al-Hilal et al. discovered associations of SNPs in the FADS gene cluster (rs174537, rs174561, rs3834458) with PUFA level [60]. Possibly, different sites at the FADS1-FADS2 locus affect the activity of the FADS1 and FADS2 enzymes. A randomized controlled study has demonstrated that an increase in the consumption of eicosapentaenoic $(20: 5 \omega 3)$ and docosahexaenoic (22:6 63 ) acids (doses of $0.45,0.9$ and $1.8 \mathrm{~g} /$ day for six months) lead to the elevation of FADS1 enzyme activity and the decrease in FADS2 activity in men and women 45-70 years old [60]. The authors of the study concluded that the rs174537 FADS variation influences FADS1 activity and the body response to the increased intake of these FAs. The study by M.A. Lankinen et al. demonstrated that FADS1-rs174550 gene polymorphism is associated with fasting blood glucose and the increased serum levels of $\omega 6$ lipid mediators synthesized from linoleic acid (18:2 $\omega 6)$ [55]. According to the available data, high linoleic acid $(18: 2 \omega 6)$

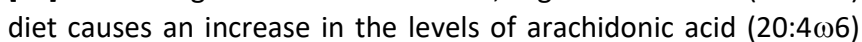
and other $\omega 6$ lipid mediators in blood, kidneys and liver in rats [61]. However, O. Coltell et al. revealed no correlations between SNPs in the FADS gene cluster and $\omega 6$ PUFA level [53].

J.Y. Bernard et al. showed that the presence of FADS1rs174546 gene in pregnant women correlated positively with $\omega 6$ PUFA level, but not with $\omega 3$ PUFA level [54]. It was established that pregnant women, carrying the minor alleles of various FADS SNPs, have high blood concentrations of PUFA precursors and low

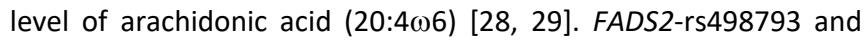
FADS1-rs174556 were demonstrated to correlate positively with

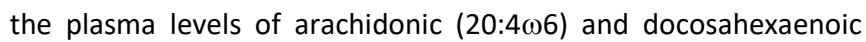
$(22: 6 \omega 3)$ acids. At the same time, there is an evidence that FADS2-

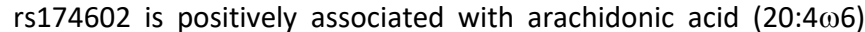
level, but correlates inversely with docosahexaenoic acid (22:6 133$)$ level [56]. These findings have also confirmed the fact that carriers of the minor allele of SNP rs174602 had a deficiency of docosahexaenoic acid (22:6 103 ).

It was also demonstrated that FADS gene polymorphisms affected breast milk concentration of PUFAs in breast-feeding women. It was discovered that FADS1-rs174556 was related to the

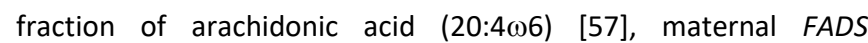
genotype was associated with the increased concentrations of linoleic acid $(18: 2 \omega 6)$ in umbilical blood, and reduced ratio of arachidonic $(20: 4 \omega 6)$ and dihomo- $\gamma$-linolenic $(20: 3 \omega 6)$ acids, as

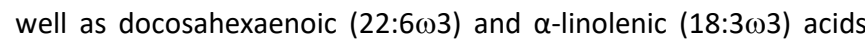
$[26,27]$. Minor allele carriage of various FADS SNPs was linked to a lower concentration of arachidonic acid $(20: 4 \omega 6)$ in breast milk [58]. Hence, FADS SNPs (FADS2-rs1535) and haplotypes (FADS2rs1535-rs3834458, FADS2-rs1535-rs174575) were associated with low concentrations of $\gamma$-linoleic $(18: 3 \omega 6)$ and arachidonic $(20: 4 \omega 6)$ acids. Similarly, the FADS haplotype (FADS1-rs174547-rs174553) was also related to declined concentrations of $\gamma$-linoleic $(18: 3 \omega 6)$ and arachidonic $(20: 4 \omega 6)$ acids in breast milk. However, the FADS2 haplotype rs3834458-rs1535-rs174575 caused an impact solely on the concentration of $\gamma$-linoleic $(18: 3 \omega 6)$, but not arachidonic $(20: 4 \omega 6)$ acid.

The association between FADS gene polymorphisms and PUFA level was found not only in adults, pregnant and breast-feeding women, but also in children. We feel that genetic and epigenetic status of maternal FADS2 gene may affect the PUFA metabolism in children. According to the published data, changes in FADS2rs174575 in a mother in combination with DNA methylation correlated with $\alpha$-linolenic acid (18:3 03 ) level in blood plasma in

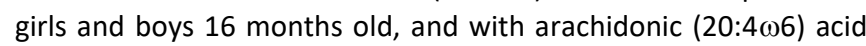
level in the group of boys [59]. Generally, changes in the FADS genes in children were associated with low concentration of 


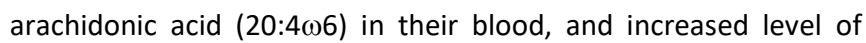

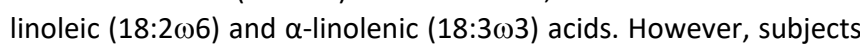
with both increased and decreased content of docosahexaenoic acid (22:6 13$)$ were noted [62]. In other studies, the association between changes in the FADS genes and docosahexaenoic acid $(22: 6 \omega 3)$ level was not observed in children above 8 years of age $[63,64]$. Children 9 months old carrying the rs174448 allele exhibited reduced levels of total cholesterol and LDL [65].

The presented results confirm an important role of the FADS genes in the regulation of PUFA levels in blood serum, kidneys, and liver [53, 61]. Genetic variations in the FADS1 and FADS2 genes involved in the $\omega 3$ PUFA metabolism may explain why the activity of these FAs is detected only in some people [25]. For example, SNPs reflecting changes in a nucleotide in the FADS1 gene alter the effectiveness of fish oil consumption in children [66].

Since polymorphisms in the FA desaturase genes affect the

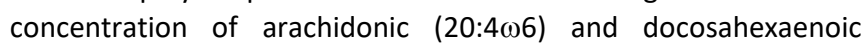
$(22: 6 \omega 3)$ acids in blood plasma, subjects with certain genotypes may require different amounts of FAs [67]. Personalized prescription of alimentary PUFAs can influence the relationship between these gene variants and FA levels [33]. Modification of the PUFA content in the diet can prevent the adverse effects of FADS gene polymorphisms [68].

The described findings suggest an important role of polymorphisms of these genes in lipid metabolism and the contribution of polymorphisms in the pathogenesis of ailments associated with lipid metabolism disorders (MS, CVDs, DM2).

\section{Polymorphisms of the FADS1 and FADS2 genes versus metabolic disorders}

\section{FADS genes versus inflammation}

Recently, it was shown that FADS1 was a regulator of inflammation [69]. $\omega 3$ and $\omega 6$ PUFAs, as well as their derivatives, play the pivotal role in onset and resolution of inflammatory reaction. M.A. Lankinen et al. reported that the FADS1 genotype influenced the relationship between the diet enriched with linoleic acid $(18: 2 \omega 6)$ and the development of inflammation [55]. The supplementation of FAs, which are the substrates for $\omega 3$ and $\omega 6$ PUFAs, to the diet of FADS1 knockout mice, was accompanied by the development of liver inflammation. The authors pointed out that inflammation was probably not linked to arachidonic acid

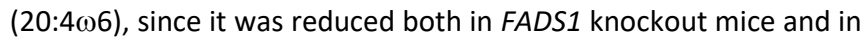
carriers of the genotype related to a reduced activity of the FADS1 enzyme. H. Hermant et al. established that FADS1-rs174547 was

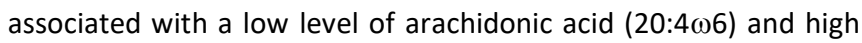

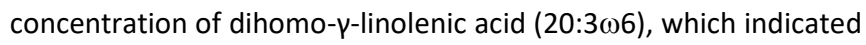
reduced FADS1 activity in carriers of this allele [70]. In addition, in FADS1 knockout mice, along with the inflammatory process in the liver, an improvement in glucose tolerance was observed [69]. It was suggested that the association between PUFAs and inflammation was mediated by eicosanoids $[5,6]$, and that relationship was modified by the FADS1 genotype. Obviously, subjects have different capacities for the synthesis of $\omega 3$ PUFAs from $\alpha$-linolenic acid and for their accumulation in erythrocytes, or tissues; therefore, the genotype can determine both high and low risk of developing chronic inflammatory diseases [33, 69].

Associations of FADS1 and FADS2 gene polymorphisms with lipid-related diseases are presented in Table 3.

\section{FADS genes versus metabolic syndrome}

Metabolic syndrome (MS) is defined as a group of metabolic disorders that are the risk factors for developing cardiovascular pathologies and type 2 diabetes [1, 10,77, 78]. The components of MS are arterial hypertension, abdominal obesity, disorders of carbohydrate metabolism, and dyslipidemia [10, 77]. Lipid changes can be both a cause and a consequence of impaired glucose metabolism and insulin resistance. Accordingly, MS and other metabolic diseases, characterized by defective glucose or lipid metabolism, are closely interrelated [79]. The association between MS and cardiovascular pathology, NAFLD, arthritis, chronic kidney disease, schizophrenia, and some types of cancer has been described [80-82].

The rs174547 polymorphism in the FADS1 gene is associated with the development of MS and its markers, such as an increased visceral fat mass and fasting hyperglycemia. Vegetarians with the FADS1-rs174547 genotype had an enhanced risk of developing MS. Decreased intake of $\alpha$-linolenic acid $(18: 3 \omega 3)$ vs. linoleic acid $(18: 2 \omega 6)$ may disrupt FA metabolism and block the formation of

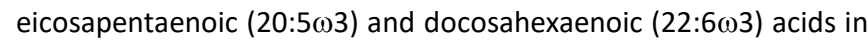
vegetarians [71].

The results of the meta-analysis by J. Dumont et al. have also confirmed the association between FADS1-rs174547 and plasma HDL level, waist circumference and body mass index (BMI). These connections were modulated by increased consumption of linoleic acid [72]. Single nucleotide polymorphisms in genes encoding FADS were discovered to relate to BMI in pregnant women [29]. Consequently, carriers of minor FADS alleles (FADS1-rs174545, FADS1-rs174546, FADS1-rs174548, FADS1-rs174553, FADS2rs1535, and FADS2-rs174583) caused a higher risk of developing obesity during pregnancy. However, women with BMI $\geq 25 \mathrm{~kg}$ before pregnancy were exposed to a lower risk of obesity in pregnancy associated with FADS polymorphisms. Overweight or obese women with SNPs in FADS2 and ELOVL2 genes had higher w3 PUFA level than normal weight women. Carriers of minor alleles of FADS1-rs174545, FADS1-rs174537, FADS1-rs174546, and FADS1-rs174553 exhibited a low ratio of arachidonic acid (20:4 $\omega 6)$ to dihomo- $\gamma$-linolenic acid (20:3 $\omega 6)$, which implied a decrease in activity of FA desaturases [29]. A.J. Yeates et al. noted high birth weights in infants as risk factor of metabolic disorders, those infants born to mothers homozygous for FADS2-rs174575 [28]. The effect of prenatal maternal docosahexaenoic acid $(22: 6 \omega 3)$ intake on infant birth weight was not unidirectional: women carrying the minor allele FADS2-rs174602 gave birth to neonates with a greater body weight than women who received placebo, which may be partly accounted for by an increase in gestational age; hence this effect requires further investigation [56].

\section{FADS genes versus type 2 diabetes mellitus}

The prevalence and mortality from DM2 continue to rise worldwide, causing important medical and social implications for the health care system [83]. This pathology is accompanied by metabolic disorders, including hypertriglyceridemia, high LDL, and low HDL.

The genetically determined FA composition and their desaturation are associated not only with lipid metabolism, but also with glucose levels and insulin secretion [30, 32]. FA desaturases play an important role in the reduction of cytosolic nicotinamide adenine dinucleotide $\left(\mathrm{NAD}^{+}\right)$to $\mathrm{NADH}$. These reactions are related to glucose metabolism. A decreased 
$\mathrm{NAD}^{+} / \mathrm{NADH}$ ratio in the cell cytosol correlates with an elevated activity of FA desaturases [84].

FADS polymorphisms can alter FA composition in plasma and modulate the development of DM2 [30, 46]. Huang M.C. et al. established that FADS1-rs174547 and FADS2-rs2727270 were associated with a decrease in the activity of FADS1/2 enzymes in patients with DM2 [32]. The effect of alimentary $\omega 3$ PUFAs in the associations between polymorphisms of the FADS genes and DM2 requires further investigation.

\section{FADS genes versus cardiovascular pathology}

The elevation of blood pressure (BP) and the prevalence of hypertension are associated with overweight and obesity in both adults and children/youth [14]. High activity of the FADS1 enzyme may protect against obesity and hypertension that are increasingly common in children and youth and tend to be observed in adulthood. In addition, the activity of this enzyme affects the

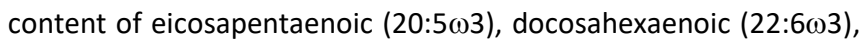

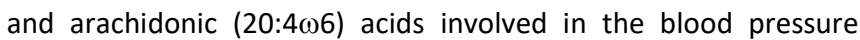

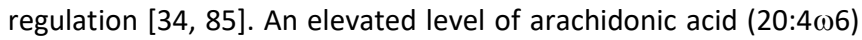
enhances blood pressure, while $\omega 3$ PUFAs reduces it [34]. FADS1 activity (assessed by the FADS1 index calculated as the ratio of arachidonic $(20: 4 \omega 6)$ to dihomo- $\gamma$-linolenic $(20: 3 \omega 6)$ acids) is inversely proportional to BMI [34] and blood pressure [86]. Since polymorphisms of the FADS1 gene are associated with PUFA content in blood plasma, the genetic component may have a substantial effect on the relationship between PUFAs and blood pressure [87].

SNP rs174546 has been shown to affect serum PUFA content [74]. Carriers of the minor allele rs174546 exhibited reduced FADS1 activity, which led to an increase in the levels of linoleic $(18: 2 \omega 6)$ and dihomo- $\gamma$-linolenic $(20: 3 \omega 6)$ acids, while arachidonic $(20: 4 \omega 6)$ and eicosapentaenoic $(20: 5 \omega 3)$ acid concentrations were reduced [74]. All haplotypes carrying the minor allele were associated with a lower FADS1 activity [74].

Increased activity of FADS2 enzyme was demonstrated in patients with coronary artery disease (CAD). In both CAD and DM2 patients, along with high FADS2 activity, the content of arachidonic $(20: 4 \omega 6)$ and dihomo- $\gamma$-linolenic $(20: 3 \omega 6)$ acids in blood plasma is increased as a result of decline in stearidonic $(18: 4 \omega 3)$ and $\alpha$-linolenic $(18: 3 \omega 3)$ acids. Simultaneously, the concentrations of eicosapentaenoic (20:5 13$)$ and

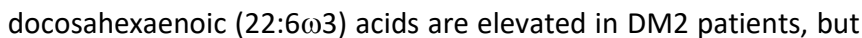
reduced in CAD patients [75].

The rs174537 G allele in the FADS gene is often detected in patients with CAD. At the same time, the rs $174537 \mathrm{G} / \mathrm{T}$ alleles in the FADS gene are associated with an elevated risk of developing this disease, while the rs $174537 \mathrm{~T}$ allele is related to the reduced risk [76]. It should be noted that DM2 patients carrying the rs174537 GG allele in the FADS gene had an augmented risk of developing CAD [76]. The carriers of the rs $174460 \mathrm{C}$ allele also had a higher risk of CAD. These results imply that the FADS gene polymorphisms affect plasma FA concentration and FA desaturase activity. Q Yang et al. studied the relationship between SNPs FADS1-rs174546 and FADS2-rs174601, and the risk of CAD and ischemic stroke (IS). The haplotype of T/T nucleotides in the FADS1 and FADS2 genes (rs174546-rs174601) was associated with an increased risk of IS, and the haplotype of $\mathrm{C} / \mathrm{C}$ nucleotides in these genes ( $r$ 174546-rs174601) was linked to a reduced risk of CAD and IS [73]. The carriers of T/T SNPs in the FADS2-rs174601 gene had a decreased HDL and apolipoprotein (ApoAI) levels in serum in the groups of patients with CAD, IS and subjects with an elevated risk of these pathologies.

The study of FADS gene polymorphisms would allow establishing the efficacy of $\omega 3$ and $\omega 6$ PUFAs in the treatment of CVDs.

\section{FADS genes versus NAFLD}

NAFLD includes a wide range of liver disorders - such as steatosis, non-alcoholic steatohepatitis (NASH), fibrosis, and cirrhosis $[88,89]$. Clinical and preclinical studies showed that NAFLD was associated with severe lipid metabolism disorders and changed FA desaturase activity (increased FADS2 activity and decreased FADS1 activity). Currently, there is no doubt that disorder of FADS activity caused by polymorphisms of their genes are accompanied by dysregulation of FA biosynthesis, which contributes to the pathogenesis of NAFLD $[4,90]$. Thus, the suppression of transcription of FADS1 genes in the liver results in an elevated risk of developing NAFLD in children [13]. Studying the relationship between FADS gene polymorphisms and the content of FADS enzymes could help in the development of personalized therapy for NAFLD. For example, children with NAFLD, who are carriers of low-functional alleles of the FADS1 genes, exhibit a better response to alimentary $\omega 3$ PUFAs [13]. Adult carriers of alleles associated with reduced FADS1 activity also respond better to w3 PUFA intake [90]. At the same time, FADS2 activity is significantly increased in patients with NAFLD combined with CAD, while this association is not observed regarding FADS2-rs3834458 polymorphism [46]. These results indicate the need for further studies of polymorphisms in genes encoding FADS for assessing the effectiveness of using $\omega 3$ PUFAs in the treatment of NAFLD and CVDs.

The FADS1/2 gene polymorphism affects the activity of FA desaturases via DNA methylation [91]. High FADS2 activity in patients with NAFLD is associated with low DNA methylation at two sites: cg06781209 and cg07999042. The cg07999042 site is linked with the risk of developing DM2 in men with a low birth weight [92]. Altered DNA methylation of several genes, in turn, is related to obesity, DM2, and NASH [93]. At the same time, impaired DNA methylation in FADS2 promotes, first of all, the change in the activity of FA desaturases and the development of hepatic steatosis, rather than inflammation or fibrosis [4, 94].

Therefore, polymorphisms of FA desaturase genes are closely linked to the disorders of lipid metabolism and diseases associated with them.

\section{Conclusion}

The literature data systematized in this review demonstrate that the impairment of the activity of FADS and their genes are accompanied by the dysregulation of the metabolic pathway involved in FA biosynthesis, which is an important component in the pathogenesis of many chronic non-communicable inflammatory diseases associated with lipid metabolism disorders. Obesity, overweight, MS, CVD, and NAFLD are typical not only for adults, but for children as well. Childhood obesity correlates with the development of CVD and DM2 in adulthood. The relationships between the risk of these pathologies and lipid metabolism disorders in pregnant and breast-feeding women are studied. The 
modulation of epigenetic mechanisms in pregnant and breastfeeding women, children and youth via using fatty acids may improve metabolic health and reduce the risk of developing lipid metabolism-related diseases in adults.

The relationship between genetic factors controlling fatty acid biosynthesis, lipid metabolism disorders, and the development of common pathologies associated with those in adults and children, was described in this review. SNP in the FADS1 gene cluster FADS1-rs174547 affect $\omega 3$ PUFA concentration in blood plasma and lead to the development of MS and DM2. FADS1-rs174546, FADS2-rs1535 SNPs also influence w3 PUFA content in blood plasma and increase the risk of obesity during pregnancy, as well as the chance of developing CAD and IS. Correlations between FADS-rs174537 and PUFA level also relate to a higher risk of CAD. FADS2-rs174602 is associated with $\omega 3, \omega 6$ PUFA levels in blood plasma and an elevated risk of metabolic disorders. Changes in FADS2-rs174575 correlate with the levels of $\omega 3$ and $\omega 6$ PUFAs in blood plasma and enhance a risk of metabolic disorders in the mother. Thus, FAs can regulate gene expression by altering epigenetic mechanisms and, therefore, they have a positive or negative effect on metabolic outcomes. Additional research is needed to detail the role of FAs in the regulation of epigenetic mechanisms in metabolic diseases. The polymorphisms of FADS1/2 genes affect the activity of FA desaturases via DNA methylation. However, it is important to investigate other epigenetic mechanisms, for example, acetylation or deacetylation of histones and miRNAs.

According to the literature, PUFAs are widely used as dietary supplements for the prevention and treatment of inflammatory diseases associated with lipid metabolism disorders. Disparities in the results of the studies assessing the benefits of PUFA supplementation may be explained by the fact that people of different ages and ethnic origins may exhibit different responses to PUFAs coming from food. Specific features of such metabolic response to the use of these FAs in adults, children, pregnant and breast-feeding women were established. Currently, many factors that may influence the ability to effectively react to alimentary PUFAs, in particular, variants of the genes involved in the PUFA metabolism, are under study.

Hence, the health effects of dietary $\omega-3$ and $\omega-6$ PUFAs may vary depending on FADS genetic variants. Further study of the genetic mechanisms regulating the metabolic pathways involved in the FA synthesis would contribute to a better understanding of the pathogenesis of many multifactorial diseases, as well as to predicting the effectiveness of their treatment via using $\omega 3$ PUFAs. Additional studies of the regulatory mechanisms of specific genes, their metabolic effects and the influence of other components (such as intestinal microbiota, methyl donors, polyphenols, etc.) on epigenetics are needed.

\section{Conflict of interest}

We declare that we have no conflicts of interest.

\section{References}

1. Jang H, Park K. Omega-3 and omega- 6 polyunsaturated fatty acids and metabolic syndrome: A systematic review and meta-analysis. Clin Nutr 2020; 39(3): 765-773. https://doi.org/10.1016/j.clnu.2019.03.032.

2. González-Becerra K, Ramos-Lopez O, Barrón-Cabrera E, Riezu-Boj Jl, Milagro FI, Martínez-López $\mathrm{E}$, et al. Fatty acids, epigenetic mechanisms and chronic diseases: A systematic review. Lipids Health Dis 2019; 18(1): 178. https://doi.org/10.1186/s12944-019-1120-6.

3. Serhan CN, Levy BD. Resolvins in inflammation: emergence of the proresolving superfamily of mediators. J Clin Invest 2018; 128: 2657-269. https://doi.org/10.1172/jci97943.

4. He Z, Zhang $R$, Jiang $F$, Zhang $H$, Zhao A, Xu B, et al. FADS1-FADS2 genetic polymorphisms are associated with fatty acid metabolism through changes in DNA methylation and gene expression. Clin. Epigenetics 2018; 10(1): 113. https://doi.org/10.1186/s13148-0180545-5.

5. Das UN. Bioactive lipids in age-related disorders. Adv Exp Med Biol 2020; 1260: 33-83. https://doi.org/10.1007/978-3-030-42667-5 3.

6. Margină D, Ungurianu A, Purdel C, Nitulescu GM, Tsoukalas D, Sarandi $E$, et al. Analysis of the intricate effects of polyunsaturated fatty acids and polyphenols on inflammatory pathways in health and disease. Food Chem Toxicol 2020; 143: 111558. https://doi.org/10.1016/j.fct.2020.111558.

7. Kytikova OY, Novgorodtseva TP, Antonyuk MV, Denisenko YK, Gvozdenko TA. Molecular targets of fatty acid ethanolamides in asthma. Medicina (Kaunas) 2019; 55(4); 87. https://doi.org/10.3390/medicina55040087.

8. Abdelhamid AS, Brown TJ, Brainard JS, Biswas P, Thorpe GC, Moore HJ, et al. Omega-3 fatty acids for the primary and secondary prevention of cardiovascular disease. Cochrane Database Syst Rev 2020; 3(2): CD003177. https://doi.org/10.1002/14651858.cd003177.pub4.

9. Watanabe $\mathrm{Y}$, Tatsuno I. Prevention of cardiovascular events with omega-3 polyunsaturated fatty acids and the mechanism involved. $J$ Atheroscler Thromb 2020; 27(3): 183-198. https://doi.org/10.5551/jat.50658.

10. Brandão $A D$, da Silva JH, Mariane Oliveira Lima S, Lima L, Loize $B$, de Castro AAM, et al. Short and long term effect of treatment nonpharmacological and lifestyle in patients with metabolic syndrome. Diabetol Metab Syndr 2020; 12: 16. https://doi.org/10.1186/s13098020-0522-y.

11. Kytikova OYu, Novgorodtseva TP, Denisenko YuK, Kovalevsky DA Metabolic and genetic determinants of lipid metabolism disruption in non-alcoholic fatty liver disease. Russian Journal of Gastroenterology, Hepatology, Coloproctology 2020; 30(2): 15-25. Russian. https://doi.org/10.22416/1382-4376-2020-30-2-15-25.

12. Kumar S, Balagopal PB. Vaspin and omentin-1 in obese children with metabolic syndrome: Two new kids on the block? Metab Syndr Relat Disord 2018; 16(2): 73-75. https://doi.org/10.1089/met.2018.0012.

13. González-Bengtsson A, Asadi A, Gao H, Dahlman-Wright K, Jacobsson A. Estrogen enhances the expression of the polyunsaturated fatty acid elongase Elovl2 via ER $\alpha$ in breast cancer cells. PLoS One 2016; 11(10): e0164241. https://doi.org/10.1371/journal.pone.0164241.

14. Martin L, Oepen J, Reinehr T, Wabitsch M, Claussnitzer G, Waldeck E, et al. Ethnicity and cardiovascular risk factors: evaluation of 40,921 normal-weight, overweight or obese children and adolescents living in Central Europe. Int J Obes (Lond) 2015; 39(1): 45-51. https://doi.org/10.1038/ijo.2014.167.

15. Ramsden CE, Makrides M, Yuan ZX, Horowitz MS, Zamora D, Yelland $\mathrm{LN}$, et al. Plasma oxylipins and unesterified precursor fatty acids are altered by DHA supplementation in pregnancy: Can they help predict risk of preterm birth? Prostaglandins Leukot Essent Fatty Acids 2020; 153: 102041. https://doi.org/10.1016/j.plefa.2019.102041.

16. Preston Mason R. New insights into mechanisms of action for omega-3 fatty acids in atherothrombotic cardiovascular disease. Curr Atheroscler Rep 2019; 21(1): 2. https://doi.org/10.1007/s11883-0190762-1.

17. Ajith TA. A recent update on the effects of omega- 3 fatty acids in Alzheimer's Disease. Curr Clin Pharmacol 2018; 13(4): 252-260. https://doi.org/10.2174/1574884713666180807145648.

18. Abdelhamid AS, Martin N, Bridges C, Brainard JS, Wang X, Brown TJ, et al. Polyunsaturated fatty acids for the primary and secondary 
prevention of cardiovascular disease. Cochrane Database Syst Rev 2018; 11(11): CD012345. https://doi.org/10.1002/14651858.cd012345.pub3.

19. Jia X, Kohli $P$, Virani SS. Omega-3 fatty acid and cardiovascular outcomes: Insights from recent clinical trials. Curr Atheroscler Rep 2019; 21(1): 1. https://doi.org/10.1007/s11883-019-0763-0.

20. Lenihan-Geels G, Bishop KS, Ferguson LR. Cancer risk and eicosanoid production: Interaction between the protective effect of long chain omega-3 polyunsaturated fatty acid intake and genotype. J Clin Med 2016; 5(2): 25. https://doi.org/10.3390/jcm5020025.

21. Denisenko YK, Kytikova OY, Novgorodtseva TP, Antonyuk MV, Gvozdenko TA, Kantur TA. Lipid-Induced mechanisms of metabolic syndrome. J Obes 2020; 2020: 5762395. https://doi.org/10.1155/2020/5762395.

22. Shama S, Liu W. Omega-3 fatty acids and gut microbiota: A reciprocal interaction in nonalcoholic fatty liver disease. Dig Dis Sci 2020; 65(3): 906-910. https://doi.org/10.1007/s10620-020-06117-5.

23. Wu JHY, Marklund $M$, Imamura $F$, Tintle $N$, Ardisson Korat $A V$, de Goede J, et al. Omega- 6 fatty acid biomarkers and incident type 2 diabetes: Pooled analysis of individual-level data for 39740 adults from 20 prospective cohort studies. Lancet Diabetes Endocrinol 2017; 5(12): 965-974. https://doi.org/10.1016/s2213-8587(17)30307-8 .

24. Harris DN, Ruczinski I, Yanek LR, Becker LC, Becker DM, Guio H, et al. Evolution of hominin polyunsaturated fatty acid metabolism: From Africa to the New World. Genome Biol Evol 2019; 11(5): 1417-1430. https://doi.org/10.1093/gbe/evz071.

25. Chilton FH, Dutta R, Reynolds LM, Sergeant S, Mathias RA, Seeds MC. Precision nutrition and omega- 3 polyunsaturated fatty acids: A case for personalized supplementation approaches for the prevention and management of human diseases. Nutrients 2017; 9(11): 1165. https://doi.org/10.3390/nu9111165.

26. Lattka E, Koletzko B, Zeilinger S, Hibbeln JR, Klopp N, Ring SM, et al. Umbilical cord PUFA are determined by maternal and child fatty acid desaturase (FADS) genetic variants in the Avon Longitudinal Study of Parents and Children (ALSPAC). Br J Nutr 2013; 109(7): 1196-1210. https://doi.org/10.1017/s0007114512003108.

27. Conway MC, McSorley EM, Mulhern MS, Strain JJ, van Wijngaarden E, Yeates AJ. Influence of fatty acid desaturase (FADS) genotype on maternal and child polyunsaturated fatty acids (PUFA) status and child health outcomes: A systematic review. Nutr Re 2020; 78(8): 627-646. https://doi.org/10.1093/nutrit/nuz086.

28. Yeates AJ, Zavez A, Thurston SW, McSorley EM, Mulhern MS, Alhamdow $A$, et al. Maternal long-chain polyunsaturated fatty acid status, methylmercury exposure, and birth outcomes in a high-fisheating mother-child cohort. J Nutr 2020; 150(7): 1749-1756. https://doi.org/10.1093/jn/nxaa131.

29. de la Garza Puentes A, Montes Goyanes R, Chisaguano Tonato AM, Torres-Espínola FJ, Arias García M, de Almeida L, et al. Association of maternal weight with FADS and ELOVL genetic variants and fatty acid levels- The PREOBE follow-up. PLoS One 2017; 12(6): e0179135. https://doi.org/10.1371/journal.pone.0179135

30. Brayner B, Kaur G, Keske MA, Livingstone KM. FADS Polymorphism, omega-3 fatty acids and diabetes risk: A systematic review. Nutrients 2018; 10(6): 758. https://doi.org/10.3390/nu10060758.

31. Czumaj A, Śledziński T. Biological role of unsaturated fatty acid desaturases in health and disease. Nutrients 2020; 12(2): 356 https://doi.org/10.3390/nu12020356.

32. Huang MC, Chang WT, Chang HY, Chung HF, Chen FP, Huang YF, et al. FADS gene polymorphisms, fatty acid desaturase activities, and HDL-C in type 2 diabetes. Int J Environ Res Public Health 2017; 14(6): 572. https://doi.org/10.3390/ijerph14060572.

33. O'Neill CM, Minihane AM. The impact of fatty acid desaturase genotype on fatty acid status and cardiovascular health in adults. Proc $\begin{array}{llll}\text { Nutr Soc 2017; } & \text { 76(1): }\end{array}$ https://doi.org/10.1017/s0029665116000732.
34. Wolters $M$, Dering $C$, Siani A, Russo $P$, Kaprio J, Risé $P$, et al. The role of a FADS1 polymorphism in the association of fatty acid blood levels, $\mathrm{BMI}$ and blood pressure in young children- Analyses based on path models. PLoS One 2017; 12(7): e0181485. https://doi.org/10.1371/journal.pone.0181485.

35. Serini S, Calviello G. Omega-3 PUFA Responders and non-responders and the prevention of lipid dysmetabolism and related diseases. Nutrients 2020; 12(5): 1363. https://doi.org/10.3390/nu12051363.

36. Zharmakhanova G, Syrlybayeva L, Nurbaulina E, Baikadamova L, Eshtayeva G. Inborn errors of fatty acid metabolism (Review). Georgian Med News 2020; (303): 161-167. Russian. https://pubmed.ncbi.nlm.nih.gov/32841199.

37. Harcombe Z. US dietary guidelines: Is saturated fat a nutrient of concern? $\mathrm{Br} J$ Sports Med 2019; 53(22): 1393-1396. https://doi.org/10.1136/bjsports-2018-099420.

38. Ko CW, Qu J, Black DD, Tso P. Regulation of intestinal lipid metabolism: Current concepts and relevance to disease. Nat Rev Gastroenterol Hepatol 2020; 17(3): 169-183. https://doi.org/10.1038/s41575-0190250-7.

39. Witard OC, Combet E, Gray SR. Long-chain n-3 fatty acids as an essential link between musculoskeletal and cardio-metabolic health in older adults. Proc Nutr Soc. 2020; 79(1): 47-55 https://doi.org/10.1017/s0029665119000922.

40. Shrestha N, Sleep SL, Cuffe JSM, Holland OJ, Perkins AV, Yau SY, et al. Role of omega- 6 and omega-3 fatty acids in fetal programming. Clin Exp Pharmacol Physiol 2020; 47(5): 907-915. https://doi.org/10.1111/1440-1681.13244.

41. Saini RK, Keum YS. Omega-3 and omega- 6 polyunsaturated fatty acids: Dietary sources, metabolism, and significance - A review. Life Sci 2018; 203: 255-267. https://doi.org/10.1016/j.Ifs.2018.04.049.

42. Tryndyak VP, Han T, Fuscoe JC, Ross SA, Beland FA, Pogribny IP. Status of hepatic DNA methylome predetermines and modulates the severity of non-alcoholic fatty liver injury in mice. BMC Genomics 2016; 17: 298. https://doi.org/10.1186/s12864-016-2617-2.

43. Castro LFC, Tocher DR, Monroig O. Long-chain polyunsaturated fatty acid biosynthesis in chordates: Insights into the evolution of Fads and Elovl gene repertoire. Prog Lipid Res 2016; 62: 25-40 https://doi.org/10.1016/j.plipres.2016.01.001.

44. Arab JP, Arrese M, Trauner M. Recent insights into the pathogenesis of nonalcoholic fatty liver disease. Annu Rev Pathol 2018; 13: 321-350. https://doi.org/10.1146/annurev-pathol-020117-043617.

45. Žák $A$, Slabý $A$, Tvrzická $E$, Jáchymová $M$, Macášek J, Vecka $M$, et al. Desaturases of fatty acids (FADS) and their physiological and clinical implication. Cas Lek Cesk 2016; 155(2): 15-21. Czech. https://pubmed.ncbi.nlm.nih.gov/27088787.

46. Jump DB, Lytle KA, Depner CM, Tripathy S. Omega-3 Polyunsaturated fatty acids as a treatment strategy for nonalcoholic fatty liver disease. Pharmacol Ther 2018; 181: 108-125. https://doi.org/10.1016/j.pharmthera.2017.07.007.

47. Joshi K, Gadgil M, Pandit A, Otiv S, Kothapalli KSD, Brenna JT. Dietary pattern regulates fatty acid desaturase 1 gene expression in Indian pregnant women to spare overall long chain polyunsaturated fatty acids levels. Mol Biol Rep 2019; 46(1): 687-693. https://doi.org/10.1007/s11033-018-4524-x.

48. Khamlaoui W, Mehri S, Hammami S, Hammouda S, Chraeif I, Elosua R, et al. Association between genetic variants in FADS1-FADS2 and ELOVL2 and obesity, lipid traits, and fatty acids in Tunisian population. Clin Appl Thromb Hemost 2020; 26: 1076029620915286. https://doi.org/10.1177/1076029620915286.

49. Rahbar E, Ainsworth HC, Howard TD, Hawkins GA, Ruczinski I, Mathias $\mathrm{R}$, et al. Uncovering the DNA methylation landscape in key regulatory regions within the FADS cluster. PLoS One 2017; 12: e0180903. https://doi.org/10.1371/journal.pone.0180903.

50. Guan W, Steffen BT, Lemaitre RN, Wu JHY, Tanaka T, Manichaikul A, et al. Genome-wide association study of plasma N6 polyunsaturated fatty 
acids within the cohorts for heart and aging research in genomic epidemiology consortium. Circ Cardiovasc Genet 2014; 7(3): 321-331. https://doi.org/10.1161/circgenetics.113.000208.

51. de Toro-Martín J, Guénard F, Rudkowska I, Lemieux S, Couture P, Vohl MC. A common variant in ARHGEF10 alters delta- 6 desaturase activity and influence susceptibility to hypertriglyceridemia. J Clin Lipidol 2018; 12(2): 311-320.e3. https://doi.org/10.1016/j.jacl.2017.10.020.

52. Tricò D, Di Sessa A, Caprio S, Chalasani N, Liu W, Liang T, et al. Oxidized derivatives of linoleic acid in pediatric metabolic syndrome: Is their pathogenic role modulated by the genetic background and the gut microbiota? Antioxid Redox Signal 2017; 30(2): 241-250. https://doi.org/10.1089/ars.2017.7049.

53. Coltell O, Sorlí JV, Asensio EM, Barragán R, González JI, Giménez-Alba $\mathrm{IM}$, et al. Genome-Wide Association study for serum omega-3 and omega- 6 polyunsaturated fatty acids: Exploratory analysis of the sexspecific effects and dietary modulation in Mediterranean subjects with metabolic syndrome. Nutrients 2020; 12(2): 310. https://doi.org/10.3390/nu12020310.

54. Bernard JY, Pan H, Aris IM, Moreno-Betancur M, Soh SE, Yap F, et al. Long-chain polyunsaturated fatty acids, gestation duration, and birth size: A Mendelian randomization study using fatty acid desaturase variants. Am J Clin Nutr 2018; 108(1): 92-100. https://doi.org/10.1093/ajcn/nqy079.

55. Lankinen MA, Fauland A, Shimizu BI, Ågren J, Wheelock CE, Laakso M, et al. Inflammatory response to dietary linoleic acid depends on FADS1 genotype. Am J Clin Nutr 2019; 109(1): 165-175. https://doi.org/10.1093/ajcn/nqy287.

56. Gonzalez-Casanova I, Rzehak P, Stein AD, Garcia Feregrino R, Rivera Dommarco JA, Barraza-Villarreal A, et al. Maternal single nucleotide polymorphisms in the fatty acid desaturase 1 and 2 coding regions modify the impact of prenatal supplementation with DHA on birth weight. Am J Clin Nutr 2016; 103(4): 1171-1178. https://doi.org/10.3945/ajcn.115.121244.

57. Mychaleckyj JC, Nayak U, Colgate ER, Zhang D, Carstensen T, Ahmed S, et al. Multiplex genomewide association analysis of breast milk fatty acid composition extends the phenotypic association and potential selection of FADS1 variants to arachidonic acid, a critical infant micronutrient. J Med Genet 2018; 55(7): 459-468. https://doi.org/10.1136/jmedgenet-2017-105134.

58. Ding Z, Liu GL, Li X, Chen XY, Wu YX, Cui CC, et al. Association of polyunsaturated fatty acids in breast milk with fatty acid desaturase gene polymorphisms among Chinese lactating mothers. Prostaglandins Leukot Essent Fatty Acids 2016; 109: 66-71. https://doi.org/10.1016/j.plefa.2016.03.009.

59. Lupu DS, Cheatham CL, Corbin KD, Niculescu MD. Genetic and epigenetic transgenerational implications related to omega-3 fatty acids. Part I: Maternal FADS2 genotype and DNA methylation correlate with polyunsaturated fatty acid status in toddlers: An exploratory analysis. Nutr Res 2015; 35(11): 939-947. https://doi.org/10.1016/j.nutres.2015.09.004.

60. Al-Hilal M, Alsaleh A, Maniou Z, Lewis FJ, Hall WL, Sanders TA, et al. Genetic variation at the FADS1-FADS2 gene locus influences delta-5 desaturase activity and LC-PUFA proportions after fish oil supplement. Lipid Res 2013; 54(2): 542-551. https://doi.org/10.1194/jlr.p032276.

61. Leng S, Winter T, Aukema HM. Dietary LA and sex effects on oxylipin profiles in rat kidney, liver, and serum differ from their effects on PUFAs. J Lipid Res 2017; 58(8): 1702-1712. https://doi.org/10.1194/jlr.m078097.

62. Harsløf LB, Larsen LH, Ritz C, Hellgren LI, Michaelsen KF, Vogel U, et al. FADS genotype and diet are important determinants of DHA status: A cross-sectional study in Danish infants. Am J Clin Nutr 2013; 97: 14031410. https://doi.org/10.3945/ajcn.113.058685

63. Lauritzen L, Sørensen LB, Harsløf LB, Ritz C, Stark KD, Astrup A, et al. Mendelian randomization shows sex-specific associations between long-chain PUFA-related genotypes and cognitive performance in
Danish schoolchildren. Am J Clin Nutr 2017; 106: 88-95. https://doi.org/10.3945/ajcn.117.152595.

64. Andersen KR, Harsløf LB, Schnurr TM, Hansen T, Hellgren LI, Michaelsen KF, et al. A study of associations between early DHA status and fatty acid desaturase (FADS) SNP and developmental outcomes in children of obese mothers. Br J Nutr 2017; 117(2): 278-286. https://doi.org/10.1017/s0007114516004645.

65. Lauritzen L, Amundsen ID, Damsgaard CT, Lind MV, Schnurr TM, Hansen T, et al. FADS and PPARG2 single nucleotide polymorphisms are associated with plasma lipids in 9-mo-old infants. J Nutr 2019; 149(5): 708-715. https://doi.org/10.1093/jn/nxy323.

66. Meldrum SJ, Li Y, Zhang G, Heaton AEM, D'Vaz N, Manz J, et al. Can polymorphisms in the fatty acid desaturase (FADS) gene cluster alter the effects of fish oil supplementation on plasma and erythrocyte fatty acid profiles? An exploratory study. Eur J Nutr 2018; 57(7): 2583-2594. https://doi.org/10.1007/s00394-017-1529-5.

67. Salas Lorenzo I, Chisaguano Tonato AM, de la Garza Puentes A, Nieto A, Herrmann F, Dieguez $E$, et al. The effect of an infant formula supplemented with $A A$ and DHA on fatty acid levels of infants with different FADS genotypes: The COGNIS study. Nutrients 2019; 11(3): 602. https://doi.org/10.3390/nu11030602.

68. Liu F, Li Z, Lv X, Ma J. Dietary n-3 Polyunsaturated fatty acid intakes modify the effect of genetic variation in fatty acid desaturase 1 on coronary artery disease. PLoS One 2015; 10: e0121255. https://doi.org/10.1371/journal.pone.0121255.

69. Gromovsky AD, Schugar RC, Brown AL, Helsley RN, Burrows AC, Ferguson D, et al. $\Delta-5$ Fatty acid desaturase FADS1 impacts metabolic disease by balancing proinflammatory and proresolving lipid mediators. Arterioscler. Thromb Vasc Biol 2018, 38(1), 218-231. https://doi.org/10.1161/ATVBAHA.117.309660.

70. Hermant X, Delay C, Flaig A, Luque-Bedregal J, Briand G, Bout MA, et al. Identification of a functional FADS1 3'UTR variant associated with erythrocyte $\mathrm{n}-6$ polyunsaturated fatty acids levels. J Clin Lipidol 2018; 12(5): 1280-1289. https://doi.org/10.1016/j.jacl.2018.07.012.

71. Ching YK, Chin YS, Appukutty M, Ramanchadran V, Yu CY, Ang GY, et al. Interaction of dietary linoleic acid and $\alpha$-linolenic acids with rs174547 in FADS1 gene on metabolic syndrome components among $\begin{array}{llll}\text { vegetarians. } \quad \text { Nutrients } & \text { 2019; } & 1686 .\end{array}$ https://doi.org/10.3390/nu11071686.

72. Dumont J, Goumidi L, Grenier-Boley B, Cottel D, Marécaux N, Montaye $M$, et al. Dietary linoleic acid interacts with FADS1 genetic variability to modulate HDL-cholesterol and obesity-related traits. Clin Nutr 2018; 37(5): 1683-1689. https://doi.org/10.1016/j.clnu.2017.07.012.

73. Yang $Q$, Yin RX, Cao XL, Wu DF, Chen WX, Zhou YJ. Association of two polymorphisms in the FADS1/FADS2 gene cluster and the risk of coronary artery disease and ischemic stroke. Int J Clin Exp Pathol 2015; 8(6): 7318-7331. https://pubmed.ncbi.nlm.nih.gov/26261632.

74. Bokor S, Dumont J, Spinneker A, Gonzalez-Gross M, Nova E, Widhalm $\mathrm{K}$, et al. Single nucleotide polymorphisms in the FADS gene cluster are associated with delta- 5 and delta- 6 desaturase activities estimated by serum fatty acid ratios. J Lipid Res 2010; 51(8): 2325-2333. https://doi.org/10.1194/jlr.m006205.

75. Li SW, Wang J, Yang Y, Liu ZJ, Cheng L, Liu HY, et al. Polymorphisms in FADS1 and FADS2 alter plasma fatty acids and desaturase levels in type 2 diabetic patients with coronary artery disease. J Transl Med 2016; 14: 79. https://doi.org/10.1186/s12967-016-0834-8.

76. Li SW, Lin K, Ma P, Zhang ZL, Zhou YD, Lu SY, et al. FADS gene polymorphisms confer the risk of coronary artery disease in a Chinese Han population through the altered desaturase activities: Based on high-resolution melting analysis. PLoS One 2013; 8(1): e55869. https://doi.org/10.1371/journal.pone.0055869.

77. Saklayen MG. The global epidemic of the metabolic syndrome. Curr Hypertens Rep 2018; 20(2): 12. https://doi.org/10.1007/s11906-018$\underline{0812-z .}$ 
78. Wang $Y$, Tu R, Yuan $H$, Shen L, Hou J, Liu X, et al. Associations of unhealthy lifestyles with metabolic syndrome in Chinese rural aged females. Sci Rep 2020; 10(1): 2718. https://doi.org/10.1038/s41598020-59607-x.

79. Niu H, Zhang H, Peng J, Wang L, Zhao X, Zhou X. et al. Quantitative endogenous peptidomics analysis of the type-2 diabetic clinical serum samples. Se Pu 2019; 37(8): 853-862. Chinese. https://doi.org/10.3724/sp.j.1123.2019.03012.

80. Lee J, Lee KS, Kim H, Jeong H, Choi MJ, Yoo HW, et al. The relationship between metabolic syndrome and the incidence of colorectal cancer. Environ Health Prev Med 2020; 25(1): 6. https://doi.org/10.1186/s12199-020-00845-w.

81. Xiang $Y$, Zhou W, Duan X, Fan Z, Wang S, Liu S, et al. Metabolic Syndrome, and particularly the hypertriglyceridemic-waist phenotype, increases breast cancer risk, and adiponectin is a potential mechanism: A case-control study in Chinese women. Front Endocrinol (Lausanne) 2019; 10: 905. https://doi.org/10.3389/fendo.2019.00905.

82. Grgurevic I, Podrug K, Mikolasevic I, Kukla M, Madir A, Tsochatzis EA. Natural history of nonalcoholic fatty liver disease: Implications for clinical practice and an individualized approach. Can J Gastroenterol Hepatol 2020; 2020: 9181368. https://doi.org/10.1155/2020/9181368.

83. Mirahmadizadeh A, Fathalipour M, Mokhtari AM, Zeighami S, Hassanipour S, Heiran A. Corrigendum to "The prevalence of undiagnosed type 2 diabetes and prediabetes in Eastern Mediterranean region (EMRO): A systematic review and metaanalysis" [Diabet. Res. Clin. Pract. 160 (2020) 107931]. Diabetes Res Clin Pract 2021; 176: 108276. Erratum for: Diabetes Res Clin Pract 2020; 160: 107931. https://doi.org/10.1016/j.diabres.2020.108276.

84. Kim W, Deik A, Gonzalez C, Gonzalez ME, Fu F, Ferrari M, et al. Polyunsaturated fatty acid desaturation is a mechanism for glycolytic NAD + recycling. Cell Metab 2019; 29(4): 856-870.e7. https://doi.org/10.1016/j.cmet.2018.12.023.

85. Ebbesson SO, Voruganti VS, Higgins PB, Fabsitz RR, Ebbesson LO, Laston $S$, et al. Fatty acids linked to cardiovascular mortality are associated with risk factors. Int J Circumpolar Health 2015; 74: 28055. https://doi.org/10.3402/ijch.v74.28055.

86. Kim SR, Jeon SY, Lee SM. The association of cardiovascular risk factors with saturated fatty acids and fatty acid desaturase indices in erythrocyte in middle-aged Korean adults. Lipids Health Dis 2015; 14 : 133. https://doi.org/10.1186/s12944-015-0135-x.

87. Schuchardt JP, Köbe T, Witte V, Willers J, Gingrich A, Tesky V, et al. Genetic variants of the FADS gene cluster are associated with erythrocyte membrane LC PUFA levels in patients with mild cognitive impairment. J Nutr Health Aging 2016; 20(6): 611-620. https://doi.org/10.1007/s12603-016-0720-3.

88. Huang TD, Behary J, Zekry A. Non-alcoholic fatty liver disease (NAFLD): A review of epidemiology, risk factors, diagnosis and management. Intern Med J 2020; 50(9): 1038-1047. https://doi.org/10.1111/imj.14709.

89. Blencowe M, Karunanayake T, Wier J, Hsu N, Yang X. Network modeling approaches and applications to unravelling non-alcoholic fatty liver disease. Genes (Basel) 2019; 10(12): 966. https://doi.org/10.3390/genes10120966.

90. Nobili V, Alisi A, Liu Z, Liang T, Crudele A, Raponi M, et al. In a pilot study, reduced fatty acid desaturase 1 function was associated with nonalcoholic fatty liver disease and response to treatment in children. Pediatr Res 2018; 84(5): 696-703. https://doi.org/10.1038/s41390018-0132-7.

91. Volkov P, Olsson AH, Gillberg L, Jørgensen SW, Brøns C, Eriksson KF, et al. A genome-wide $\mathrm{mQTL}$ analysis in human adipose tissue identifies genetic variants associated with DNA methylation, gene expression and metabolic traits. PLoS One 2016; 11(6): e0157776. https://doi.org/10.1371/journal.pone.0157776.

92. Perfilyev A, Dahlman I, Gillberg L, Rosqvist F, Iggman D, Volkov P, et al. Impact of polyunsaturated and saturated fat overfeeding on the DNAmethylation pattern in human adipose tissue: a randomized controlled trial. Am J Clin Nutr 2017; 105(4): 991-1000. https://doi.org/10.3945/ajcn.116.143164.

93. Vaittinen $M$, Walle $P$, Kuosmanen E, Mannisto V, Kakela P, Agren J, et al. FADS2 genotype regulates delta-6 desaturase activity and inflammation in human adipose tissue. J Lipid Res 2016; 57(1): 56-65. https://doi.org/10.1194/jlr.m059113.

94. Gillberg L, Perfilyev A, Brøns C, Thomasen M, Grunnet LG, Volkov P, et al. Adipose tissue transcriptomics and epigenomics in low birthweight men and controls: Role of high-fat overfeeding. Diabetologia 2016; 59(4): 799-812. https://doi.org/10.1007/s00125-015-3852-9.

\section{Authors:}

Oksana Yu. Kytikova - MD, DSc, Researcher, Laboratory of Rehabilitative Treatment, Vladivostok Branch of Far Eastern Scientific Center of Respiratory Physiology and Pathology, Research Institute of Medical Climatology and Rehabilitative Treatment, Vladivostok, Russia. http://orcid.org/0000-0001-5018-0271.

Tatyana P. Novgorodtseva - DSc, Professor, Senior Researcher, Laboratory of Biomedical Research, Vladivostok Branch of Far Eastern Scientific Center of Respiratory Physiology and Pathology, Research Institute of Medical Climatology and Rehabilitative Treatment, Vladivostok, Russia. http://orcid.org/0000-0002-6058-201x.

Yulia K. Denisenko - DSc, Head of the Laboratory of Biomedical Research, Vladivostok Branch of Far Eastern Scientific Center of Respiratory Physiology and Pathology, Research Institute of Medical Climatology and Rehabilitative Treatment, Vladivostok, Russia. https://orcid.org/00000003-4130-8899.

Marina V. Antonyuk - MD, DSc, Professor, Head of the Laboratory of Rehabilitative Treatment, Vladivostok Branch of Far Eastern Scientific Center of Respiratory Physiology and Pathology, Research Institute of Medical Climatology and Rehabilitative Treatment, Vladivostok, Russia. https://orcid.org/0000-0002-2492-3198.

Tatyana A. Gvozdenko - MD, DSc, Professor of Russian Academy of Sciences; Senior Researcher, Laboratory of Rehabilitative Treatment, Vladivostok Branch of Far Eastern Scientific Center of Respiratory Physiology and Pathology, Research Institute of Medical Climatology and Rehabilitative Treatment, Vladivostok, Russia. http://orcid.org/0000-00026413-9840. 